\title{
Total Generalized Variation Method for Deconvolution-based CT Brain Perfusion
}

\author{
D.A. Lyukov ${ }^{1}$, A.S. Krylov ${ }^{1}$, V.A. Lukshin ${ }^{2}$ \\ d@lyukov.com|kryl@cs.msu.ru|wlukshin@nsi.ru \\ ${ }^{1}$ Laboratory of Mathematical Methods of Image Processing, Faculty of Computational Mathematics and Cybernetics, \\ Lomonosov Moscow State University, Moscow, Russia; \\ ${ }^{2}$ National Medical Research Center of Neurosurgery named after academician N.N. Burdenko, Moscow, Russia \\ Deconvolution-based method for image analysis of cerebral blood perfusion computed tomography has been \\ suggested. This analysis is the important part of diagnostics of ischemic stroke. The method is based on total \\ generalized variation regularization algorithm. The algorithm was tested with generated synthetic data and clinical \\ data. Proposed algorithm was compared with singular value decomposition method using Tikhonov regularization \\ and with total variation based deconvolution method. It was shown that the suggested algorithm gives better results \\ than these methods. The proposed algorithm combines both deconvolution and denoising processes, so results are \\ more noisy resistant. It can allow to use lower radiation dose.
}

Keywords: computed tomography, cerebral perfusion, deconvolution method, total generalized variation.

\section{Introduction}

Cerebral perfusion computer tomography is an important method for ischemic stroke diagnostics. This test can allow to localize the area of brain damaged by stroke. It is very important for urgent surgery. Usually this information is obtained using method based on the iodinated contrast agent injection and CT scans of its concentration [1]. Example of such CT scan is shown in Fig. 1.

The basic characteristics of cerebral blood dynamics are cerebral blood flow (CBF), cerebral blood volume (CBV) and mean transit time (MTT), calculating at each point of brain.

There are nondeconvolution-based methods of calculation of these characteristics: moment method and method of maximum slope $[2,3]$.

Better results can be obtained using deconvolution-based methods. Total variation based deconvolution method was suggested in $[4,5]$. Nevertheless total variation approach leads to some artifacts, because it tends to make solution piece-wise constant. In this paper we suggest a deconvolution approach using total generalized variation regularization. In this case solution has not piece-wise constant artifacts.

Quality of CT scans depends on the radiation dose: the higher the radiation, the higher the image quality. Thus, the noisy resistant method can allow to use lower radiation dose.

One of the approaches to reducing the noise impact is the denoising of perfusion scans before deconvolution or perfusion maps after deconvolution [6]. The method suggested in our paper includes denoising stage inside the deconvolution procedure. It decreases the number of method parameters and make the results more stable.

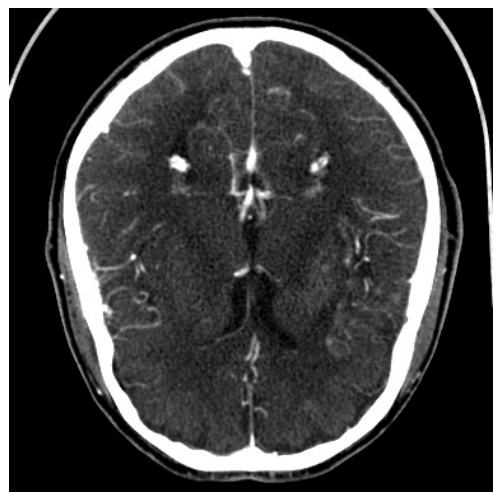

Fig. 1. An example of CT scan at one point in time.

\section{Theoretical model}

We consider some area of brain, where function $c_{\text {tissue }}(t, x, y)$ is defined. This function is the concentration of contrast agent obtained from intensity of CT scans at point $(x, y)$. Concentration in artery point is considered as an artery input function (AIF). We denote it by $c_{\text {artery }}(t)$.

These functions are connected by the relation given by the convolution equation $[2,3]$ :

$$
\begin{aligned}
c_{\text {tissue }}(t, x, y) & =\left(c_{\text {artery }} * k\right)(t) \\
& =\int_{-\infty}^{+\infty} c_{\text {artery }}(\xi) k(t-\xi, x, y) d \xi
\end{aligned}
$$

where $k(t, x, y)$ is a residual function at point $(x, y)$.

Characteristics CBF, CBV and MTT at point $(x, y)$ can be represented via residual function $k(t)$ [3]:

$$
\begin{aligned}
& C B F=\frac{1}{\rho_{\text {tissue }}} \max k(t), \\
& C B V=\frac{1}{\rho_{\text {tissue }}} \int_{0}^{\infty} k(\tau) d \tau, \\
& M T T=\frac{1}{\max k(t)} \int_{0}^{\infty} k(\tau) d \tau,
\end{aligned}
$$

where $\rho_{\text {tissue }}$ is a constant density of tissue.

We consider all functions on finite uniform grid, so equation (1) turns to linear system: 


$$
\mathbf{A k}=\mathbf{c}
$$

where:

$\mathbf{A}=\Delta t\left(\begin{array}{cccc}c_{\text {artery }}\left(t_{1}\right) & 0 & \ldots & 0 \\ c_{\text {artery }}\left(t_{2}\right) & c_{\text {artery }}\left(t_{1}\right) & \ldots & 0 \\ \vdots & \vdots & \ddots & \vdots \\ c_{\text {artery }}\left(t_{T}\right) & c_{\text {artery }}\left(t_{T-1}\right) & \ldots & c_{\text {artery }}\left(t_{1}\right)\end{array}\right)$

There $t_{1}, t_{2}, \ldots, t_{T}$ are the nodes of the uniform grid with step $\Delta t$.

\section{Deconvolution problem}

Solution of the deconvolution problem for equation (3) is an ill-posed problem, and matrix $\mathbf{A}$ is illconditioned. If we solve system (3) directly, solution $\mathbf{k}$ will be unstable. Small noise in input data can completely change solution and the solution can not be used for medical diagnosis.

An example of function $k(t)$ obtained without regularization procedure for system (3) is shown in Fig. $2-3$. The unstability is so large that we can not find any real information on the solution.

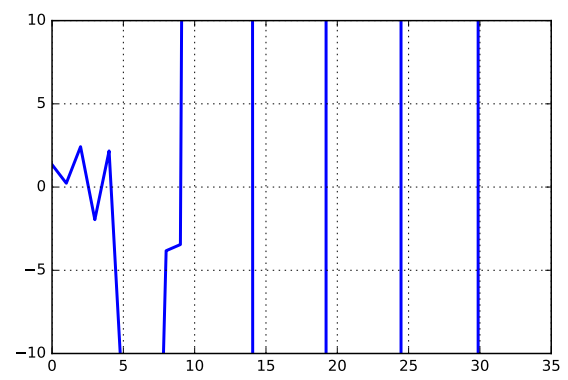

Fig. 2. $k(t)$. Range is limited to interval $[-10,10]$, so bigger oscillations are seen as vertical strips.

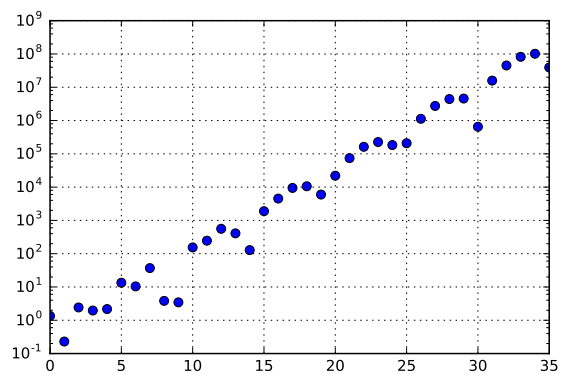

Fig. 3. $|k(t)|$ on a logarithmic scale.

There are different methods to regularize the problem. In this paper we consider method of total generalized variation and compare it with some state-ofthe-art methods.

\subsection{Singular value decomposition (SVD)}

We represent matrix $\mathbf{A}$ as a product of three matrices [7]:

$$
\mathbf{A}=\mathbf{U} \Sigma \mathbf{V}^{\mathbf{T}},
$$

where $\mathbf{U}$ and $\mathbf{V}$ are orthogonal matrices, and

$$
\Sigma=\operatorname{diag}\left[\sigma_{1}, \sigma_{2}, \ldots, \sigma_{r}\right]
$$

is a diagonal matrix composed of the singular values of matrix $\mathbf{A}, r=\operatorname{rang} \mathbf{A}$.

This representation is called singular value decomposition (SVD) of matrix A.

Using SVD, we can write the solution of (3) in the form:

$$
\mathbf{k}_{\mathbf{l s}}=\mathbf{V} \Sigma^{-1} \mathbf{U}^{\mathbf{T}} \mathbf{c}
$$

where $\Sigma^{-1}=\operatorname{diag}\left[\sigma_{1}^{-1}, \sigma_{2}^{-1}, \ldots, \sigma_{r}^{-1}\right]$.

It is the solution of following minimization problem:

$$
\mathbf{k}_{\mathbf{l s}}=\underset{\mathbf{k} \in \mathbb{R}^{T}}{\arg \min }\left(\|\mathbf{A} \mathbf{k}-\mathbf{c}\|_{2}^{2}\right) .
$$

Small singular values make a huge impact on the values of $\mathbf{k}$. It is the reason of ill-conditioning of matrix $\mathbf{A}$. These values can be suppressed using smoothing factor $\lambda$ :

$$
\sigma_{i, \lambda}^{(t i k h)}=\frac{\sigma_{i}}{\sigma_{i}^{2}+\lambda^{2}} .
$$

Using of matrix

$$
\Sigma_{\lambda}^{-1}=\operatorname{diag}\left[\sigma_{1, \lambda}^{(t i k h)}, \sigma_{2, \lambda}^{(t i k h)}, \ldots, \sigma_{r, \lambda}^{(t i k h)}\right]
$$

instead of $\Sigma^{-1}$ in (6), we get another method called Tikhonov regularization. Here $\lambda$ is a regularization parameter. Vector $\mathbf{k}_{\lambda}^{(t i k h)}$ obtained by this method is the solution of another minimization problem:

$$
\mathbf{k}_{\lambda}^{(t i k h)}=\underset{\mathbf{k} \in \mathbb{R}^{T}}{\arg \min }\left(\|\mathbf{A k}-\mathbf{c}\|_{2}^{2}+\lambda^{2}\|\mathbf{k}\|_{2}^{2}\right) .
$$

\subsection{Total variation (TV)}

Let consider matrices composed by values of $\mathbf{k}$ and $\mathbf{c}$ in all considering points of space:

$$
\mathbf{K}=\left[\mathbf{k}_{\mathbf{1}}, \mathbf{k}_{\mathbf{2}}, \ldots, \mathbf{k}_{\mathbf{N}}\right], \mathbf{C}=\left[\mathbf{c}_{\mathbf{1}}, \mathbf{c}_{\mathbf{2}}, \ldots, \mathbf{c}_{\mathbf{N}}\right] .
$$

Generally, regularization method in this paper can be written as a minimization problem of functional:

$$
J(\mathbf{K})=F(\mathbf{K}, \mathbf{C})+R(\mathbf{K}, \lambda),
$$

where $F(\mathbf{K}, \mathbf{C})$ is the data fidelity functional, $R(\mathbf{K}, \lambda)$ is regularization term, and $\lambda$ is a regularization parameter.

The most common used data fidelity functional is the Frobenius norm of the residual:

$$
F(\mathbf{K}, \mathbf{C})=\|\mathbf{A K}-\mathbf{C}\|_{2}^{2} .
$$

In total variation method we use following regularization functional $[4,5]$ :

$$
\begin{aligned}
R(\mathbf{K}, \lambda)=\|\mathbf{K}\|_{T V}^{\lambda} & =\sum_{i, j, t} \lambda_{1}\left|\widetilde{\mathbf{K}}_{i+1, j, t}-\widetilde{\mathbf{K}}_{i, j, t}\right| \\
& +\sum_{i, j, t} \lambda_{1}\left|\widetilde{\mathbf{K}}_{i, j+1, t}-\widetilde{\mathbf{K}}_{i, j, t}\right| \\
& +\sum_{i, j, t} \lambda_{2}\left|\widetilde{\mathbf{K}}_{i, j, t+1}-\widetilde{\mathbf{K}}_{i, j, t}\right|
\end{aligned}
$$


where $\widetilde{\mathbf{K}} \in \mathbb{R}^{N_{1} \times N_{2} \times T}$ is the reshaped matrix $\mathbf{K}$, $\lambda=\left(\lambda_{1}, \lambda_{2}\right)$ is a regularization parameter.

We can use different weights for spatial and temporal derivatives.

Total variation approach may lead to some artifacts, because it makes solution a piece-wise constant.

\subsection{Total generalized variation (TGV)}

Total generalized variation uses also the second order derivative. In this work we use following form of TGV stabilizer [8]:

$$
T G V_{\lambda}^{2}(z)=\lambda_{1}\|\nabla z\|_{1}+\lambda_{2}\|\nabla(\nabla z)\|_{1} .
$$

Approximation on regular grid for one-dimensional case can be written as

$$
\begin{aligned}
T G V_{\lambda}^{2}(z) & =\lambda_{1} \sum_{i}\left|z_{i+1}-z_{i}\right| \\
& +\lambda_{2} \sum_{i}\left|z_{i+1}-2 z_{i}+z_{i-1}\right|,
\end{aligned}
$$

where $z=\left(z_{1}, z_{2}, \ldots, z_{T}\right)$ is the grid function.

Finally, the regularization functional has the form:

$$
\begin{aligned}
R(\mathbf{K}, \lambda) & =\sum_{i, j, t} \lambda_{1}\left|\widetilde{\mathbf{K}}_{i+1, j, t}-\widetilde{\mathbf{K}}_{i, j, t}\right| \\
& +\sum_{i, j, t} \lambda_{2}\left|\widetilde{\mathbf{K}}_{i+1, j, t}-2 \widetilde{\mathbf{K}}_{i, j, t}+\widetilde{\mathbf{K}}_{i-1, j, t}\right| \\
& +\sum_{i, j, t} \lambda_{1}\left|\widetilde{\mathbf{K}}_{i, j+1, t}-\widetilde{\mathbf{K}}_{i, j, t}\right| \\
& +\sum_{i, j, t} \lambda_{2}\left|\widetilde{\mathbf{K}}_{i, j+1, t}-2 \widetilde{\mathbf{K}}_{i, j, t}+\widetilde{\mathbf{K}}_{i, j-1, t}\right| \\
& +\sum_{i, j, t} \lambda_{3}\left|\widetilde{\mathbf{K}}_{i, j, t+1}-\widetilde{\mathbf{K}}_{i, j, t}\right| \\
& +\sum_{i, j, t} \lambda_{4}\left|\widetilde{\mathbf{K}}_{i, j, t+1}-2 \widetilde{\mathbf{K}}_{i, j, t}+\widetilde{\mathbf{K}}_{i, j, t-1}\right| .
\end{aligned}
$$

where $\lambda=\left(\lambda_{1}, \lambda_{2}, \lambda_{3}, \lambda_{4}\right)$ is a regularization parameter.

\section{Optimization algorithm}

We use Nesterov accelerated gradient descent [9] for functional minimization (9):

$$
\begin{aligned}
z_{0} & =0, \\
y_{0} & =0, \\
z_{k+1} & =y_{k}-\epsilon \cdot \nabla F\left(y_{k}\right), \\
y_{k+1} & =z_{k}+\beta_{k}\left(z_{k+1}-z_{k}\right),
\end{aligned}
$$

where $\beta_{k}=1-3 /(k+1)$, and $\epsilon$ is the learning rate. In this paper we used $\epsilon=10^{-8}$.

\section{Method testing}

Clinical perfusion data does not have ground truth values of residue function $k(t)$ and perfusion parameters. Therefore, synthetic data was generated. As a base for generation we take perfusion maps for phantom [10]. Then we generate function $k(t)=C \cdot e^{-(a t)^{2}}$.
To evaluate the stability of the method, we add a white additive gaussian noise to the synthetic data.

Residue function $k(t)$ - the result of applying of described methods - is shown in Fig. 4.

We do not have ground truth values for clinical data, but we can compare methods in Fig. 4. We can see that residue function $k(t)$ with TGV does not tends to be a piece-wise constant as it is with TV method.

The obtained mean transit time (MTT) map for synthetic data by different method is given in Fig. 5 . MTT map is most informative for diagnosis purposes.

\section{References}

[1] Axel Leon. Cerebral blood flow determination by rapid-sequence computed tomography: theoretical analysis. // Radiology. - 1980. - Vol. 137, no. 3. - P. 679-686.

[2] Theoretic basis and technical implementations of CT perfusion in acute ischemic stroke, part 1: theoretic basis / A.A. Konstas, G.V. Goldmakher, TY Lee, M.H. Lev // American Journal of Neuroradiology. - 2009. - Vol. 30, no. 4. - P. 662-668.

[3] Deconvolution-based CT and MR brain perfusion measurement: theoretical model revisited and practical implementation details / Andreas Fieselmann, Markus Kowarschik, Arundhuti Ganguly et al. // Journal of Biomedical Imaging. — 2011. Vol. 2011. - P. 14.

[4] Tensor total-variation regularized deconvolution for efficient low-dose CT perfusion / Ruogu Fang, Pina C Sanelli, Shaoting Zhang, Tsuhan Chen // International Conference on Medical Image Computing and Computer-Assisted Intervention / Springer. - 2014. - P. 154-161.

[5] Robust low-dose CT perfusion deconvolution via tensor total-variation regularization / Ruogu Fang, Shaoting Zhang, Tsuhan Chen, Pina C Sanelli // IEEE transactions on medical imaging. - 2015. - Vol. 34, no. 7. - P. 1533-1548.

[6] Kadimesetty V. S. et al. Convolutional neural network-based robust denoising of low-dose computed tomography perfusion maps / / IEEE Transactions on Radiation and Plasma Medical Sciences. - 2018. - Vol. 3. - no. 2. - P. 137-152.

[7] Golub Gene H., Reinsch Christian. Singular value decomposition and least squares solutions // Numerische mathematik. - 1970. — Vol. 14, no. 5. - P. 403-420.

[8] Nasonov A., Krylov A. An improvement of BM3D image denoising and deblurring algorithm by generalized total variation // 2018 7th European Workshop on Visual Information Processing (EUVIP). - 2018. - P. 1-4.

[9] Bottou L., Curtis F. E., Nocedal J. Optimization methods for large-scale machine learning // Siam Review. - 2018. - Vol. 60. - no. 2. - P. 223-311. 

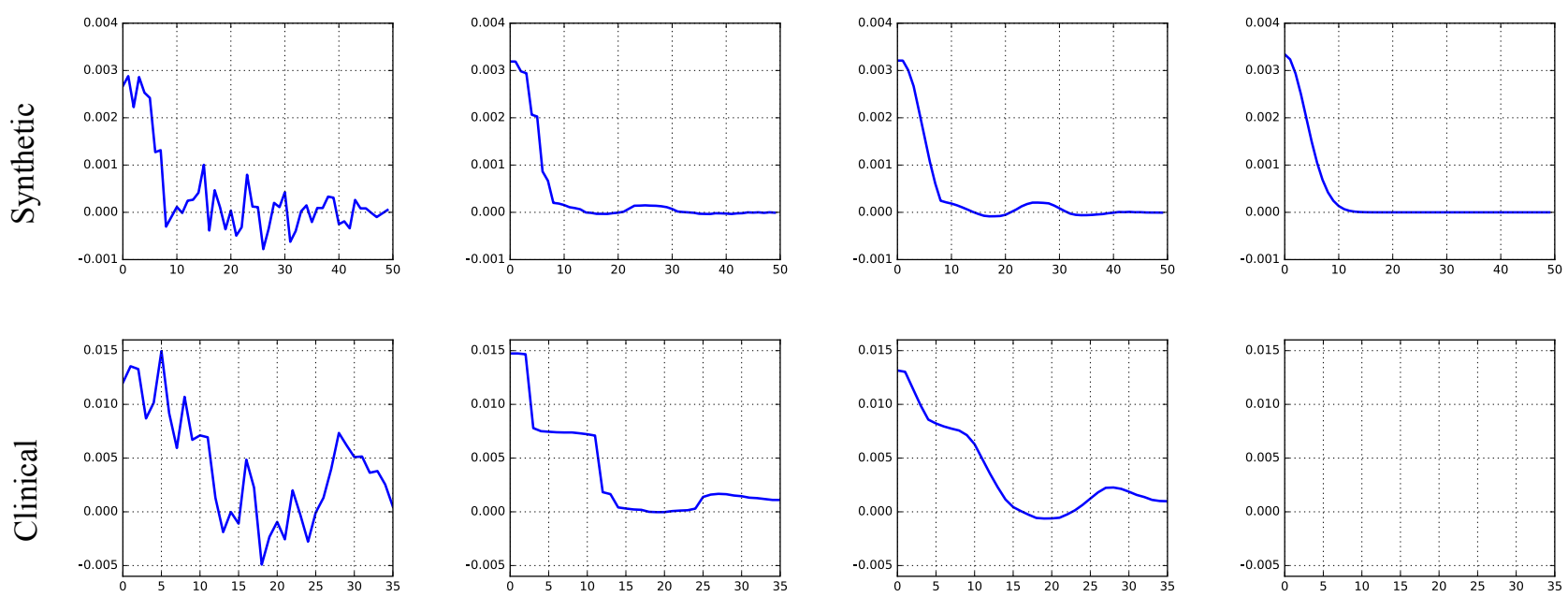

SVD

TV

TGV

Ground truth

Fig. 4. $k(t)$ for a brain tissue point.

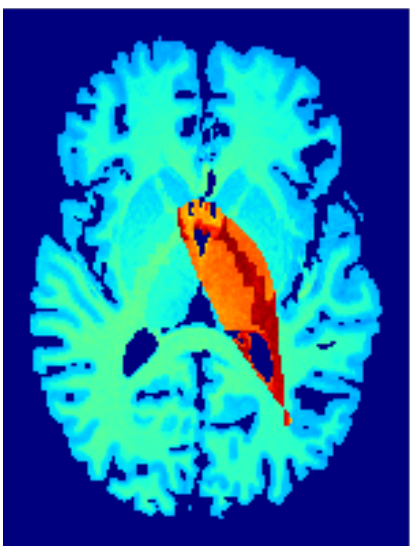

(a) Ground truth

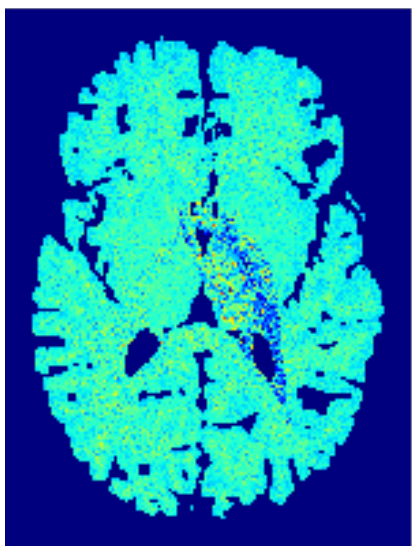

(b) SVD

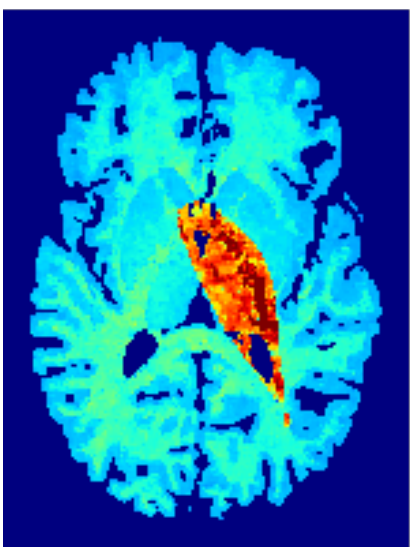

(c) TV

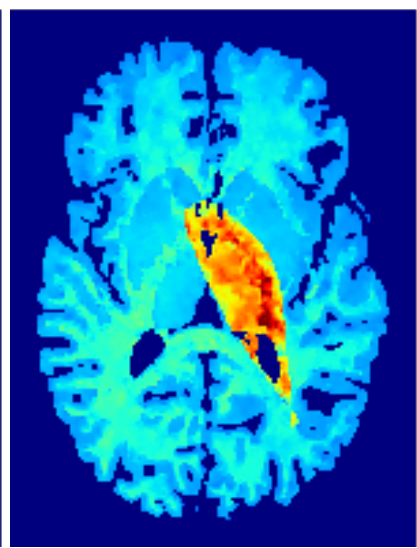

(d) TGV

Fig. 5. MTT results for synthetic data by different methods.

[10] Digital brain perfusion phantom. https://www5.cs.fau.de/research/data/digitalbrain-perfusion-phantom/. 